'Servicio de Medicina, Sección Nefrología, Hospital Naval A. Nef, Viña del Mar, Chile.

2Servicio de Medicina, Sección Nefrología, Hospital Dr. Gustavo Fricke, Viña del Mar, Chile.

${ }^{3}$ Departamento de Medicina, Escuela de Medicina, Universidad de Valparaíso, Valparaíso, Chile. ${ }^{4}$ Escuela de Medicina, Sede Viña del Mar, Universidad Andrés Bello, Viña del Mar, Chile.

${ }^{5}$ Servicio de Medicina, Sección Infectología, Hospital Naval A. Nef, Viña del Mar, Chile.

${ }^{6}$ Departamento de Anatomía Patológica, Facultad de Medicina, Pontificia Universidad Católica de Chile, Santiago, Chile.

Recibido el 15 de febrero de 2016, aceptado el 13 julio de 2016.

Correspondencia a: Dr. Jorge Vega 5 Norte 1035, Viña del Mar, Chile.

Teléfono: 56-32-2974237 jvegastieb@gmail.com

\section{Diagnóstico de infección por VIH por el hallazgo incidental de alteraciones ultraestructurales en una biopsia renal}

\author{
JORGE VEGA ${ }^{1,2,3}$, M. ELIZABETH BARTHEL ${ }^{4,5}$, GONZALO P. MÉNDEZ ${ }^{6}$
}

HIV infection has different clinical presentations. We report a 21-year-old male with longstanding isolated microscopic hematuria attributed to thin glomerular basement membrane disease, who after 15 years of follow-up presented with significant proteinuria. A kidney biopsy was performed, revealing the presence of tubulo-reticular inclusions in the glomerular endothelial cells. This finding led to suspect an HIV infection, which was verified. Antiretroviral therapy, angiotensin-converting enzyme and angiotensin II receptor blockers were prescribed. At 6 years of diagnosis the patient is asymptomatic and has normal kidney function. Microscopic hematuria and low level proteinuria persists.

(Rev Med Chile 2016; 144: 1218-1221)

Key words: AIDS-Associated Nephropathy; Hematuria; HIV; Thin Membrane Nephropathy.

L a infección por el virus de inmunodeficiencia humana (VIH) puede presentarse clínicamente de formas muy diversas. Inicialmente se sospechaba frente a infecciones oportunistas, fiebre de etiología desconocida, enflaquecimiento involuntario, tumores (sarcoma de Kaposi, linfomas), etc.

Actualmente es frecuente diagnosticarla por exámenes realizados a tuberculosos, donantes de sangre, evaluaciones de salud o en la investigación de otras enfermedades (glomerulopatías, colestasias, infiltrados pulmonares, exantemas cutáneos, disfunciones neurológicas, etc. $)^{1}$.

La infección por VIH puede producir alteraciones histológicas que pueden ser observadas casualmente en biopsias efectuadas por otros motivos, permitiendo el diagnóstico antes de la aparición de las primeras manifestaciones clínicas².

El motivo de esta comunicación es la presentación de un caso de infección asintomática por VIH descubierta por el hallazgo casual de alteraciones ultraestructurales en una biopsia renal, efectuada para la evaluación de una glomerulopatía.

\section{Caso clínico}

Hombre caucásico, obeso (IMC: $30,7 \mathrm{~kg} / \mathrm{m}^{2}$ ), con antecedentes de jaqueca e hiperlipidemia. Consultó a los 21 años para un examen de salud. Estaba asintomático y sólo destacaba su obesidad. En los exámenes había leve elevación de transaminasas e hipertrigliceridemia. La glicemia, uremia, creatinina, colesterol total, hemograma y VHS eran normales. El análisis de orina mostró hematuria microscópica (50-60 por campo), sin otras alteraciones. La ecotomografía abdominal informó esteatosis hepática y riñones de aspecto normal. La pielografía endovenosa no mostró alteraciones. Clearance de creatinina: $102 \mathrm{ml} /$ $\mathrm{min} / 1,73 \mathrm{~m}^{2}$, creatinina: $0,98 \mathrm{mg} / \mathrm{dl}$, presión arterial: 120/80 mmHg. Un segundo examen de orina 


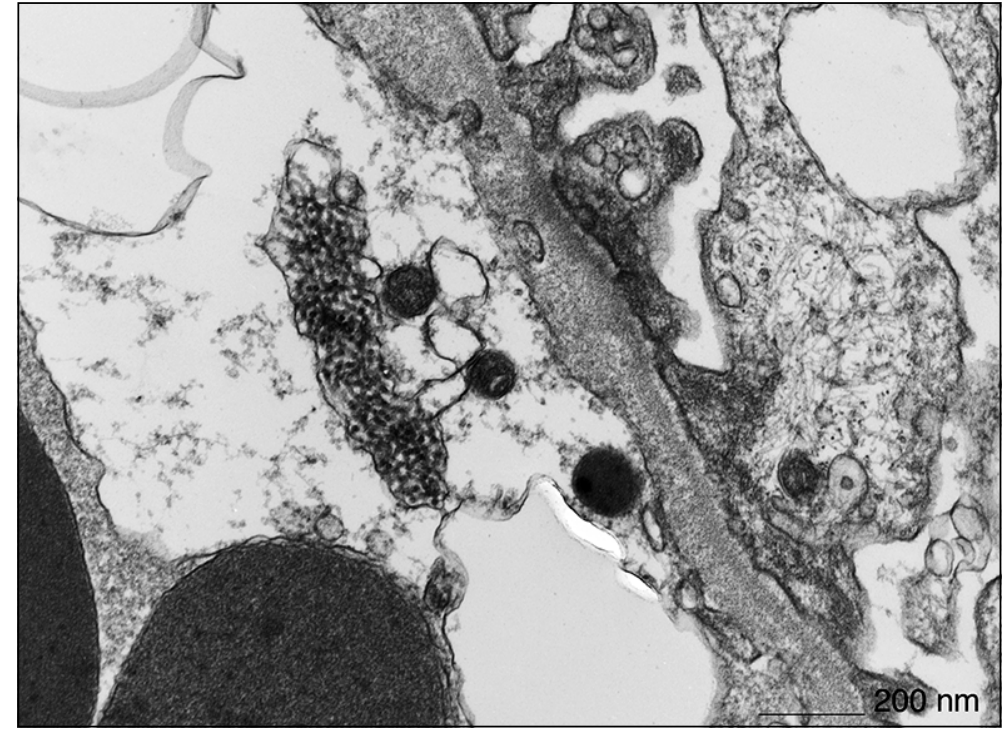

Figura 1. Microscopia electrónica de transmisión. Segmento de asa capilar glomerular que muestra su membrana basal muy delgada, con menos de $100 \mathrm{~nm}$ de espesor en la lamina densa y con ensanchamiento de procesos pedicelares adyacentes. Al centro de la imagen se reconoce una gran inclusión túbulo-reticular endotelial (tinción de acetato de uranilo y citrato de plomo; 16.000x). mostró hematuria sin proteinuria detectable. $\mathrm{Su}$ madre presentaba hematuria microscópica por años. Se planteó una probable enfermedad de la membrana basal delgada glomerular (EMBD) y un hígado graso. Se aconsejó bajar de peso y observar la evolución de la hematuria. En los 14 años siguientes, se controló regularmente persistiendo la hematuria microscópica (se observaron ocasionalmente cilindros hemáticos), en ausencia de proteinuria o deterioro funcional renal. $\mathrm{Al}$ año 15 de control la hematuria persistía, clearance de creatinina $199 \mathrm{ml} / \mathrm{min} / 1,73 \mathrm{~m}^{2}$, perfil lipídico normal, apareciendo proteinuria significativa (1.776 $\mathrm{mg} / 24 \mathrm{~h} ; 89 \%$ albúmina) y cilindros grasos. Ante esta evolución atípica de una EMBD se efectuó una biopsia renal (BpR).

El examen de microscopia óptica mostró un tejido renal sin signos de daño crónico; contuvo 7 glomérulos, todos con signos de hipertrofia, pero sin alteraciones de su arquitectura mesangiocapilar y signos tubulares de proteinuria. La inmunofluorescencia no evidenció depósitos de inmunoglobulinas ni complemento en glomérulos. El examen de microscopia electrónica confirmó la ausencia de depósitos electrón-densos, destacando el adelgazamiento casi difuso de la membrana basal glomerular $(80 \%$ del perímetro capilar), promediando $170 \mathrm{~nm}$ en las mediciones seriadas, acompañada de desaparición pedicelar intensa (80\%). Las células endoteliales contenían inclusiones túbulo-reticulares (ITR) (Figura 1). Por la aparición de ITR se solicitó serología para enfermedades colágeno-vasculares, HBsAg, HVC y VIH; resultando esta última positiva. El recuento de CD4 fue 1.318 Linf/uL-38\% y carga viral 2.800 copias ARN $/ \mathrm{ml}$. Se plantearon los diagnósticos de EMBD e infección por VIH. Se prescribió telmisar$\tan 40 \mathrm{mg} /$ día, zidovudina, lamivudina y efavirenz. El paciente relató haber tenido un contacto sexual no protegido reciente.

$\mathrm{Al}$ año siguiente, la proteinuria era $2.000 \mathrm{mg} /$ día con empeoramiento de la hiperlipidemia. Se adicionó lisinopril $20 \mathrm{mg}$ y rosuvastatina $20 \mathrm{mg}$. Los años siguientes transcurrieron sin eventos importantes salvo serología (+) para sífilis que se trató con penicilina.

Seis años después de la BpR el paciente permanecía asintomático, la presión arterial y la función renal eran normales, persistía la hematuria microscópica, la proteinuria era moderada $(680 \mathrm{mg} / 24$ h), la carga viral era indetectable y el recuento de CD4 1.140 por $\mathrm{mm}^{3}$.

\section{Discusión}

En este paciente, la presencia de ITR en las células endoteliales glomerulares en la BpR, indicada por la aparición de proteinuria significativa durante la evolución de una presunta EMBD, hizo sospechar una infección por VIH. 
Las ITR son estructuras intracelulares observadas mediante microscopía electrónica principalmente en el citoplasma de células endoteliales $y$ linfocitos ${ }^{3}$. Se han visualizado en órganos como riñón, pulmón, hígado, piel, músculos y ojos ${ }^{4,5}$.

Las ITR son subestructuras de $23-25 \mathrm{~nm}$ dispuestas como pequeños racimos de microtúbulos anastomosados que se encuentran asociados a las cisternas del retículo endoplásmico liso ${ }^{4,6}$. También pueden observarse en el citoplasma, en los espacios perinucleares y en el núcleo de las células ${ }^{4}$. Su presencia es un marcador de la estimulación sistémica por interferon (IFN) ${ }^{3}$. Las ITR se forman en respuesta a concentraciones elevadas de IFN alfa y beta endógenos o al uso de IFN alfa y gamma exógenos $^{2,7}$. El número de ITR es proporcional a las concentraciones de IFN y a la duración de su exposición ${ }^{7}$.

La presencia de ITR en BpR se observa mayoritariamente en las células endoteliales glomerulares de pacientes aquejados de enfermedades colágeno-vasculares o infecciones virales ${ }^{8}$. Las más frecuentes son el lupus eritematoso sistémico (LES) y la infección por $\mathrm{VIH}^{8}$. Con menos frecuencia, las ITR se han observado en dermatomiositis, polimiositis, esclerodermia, síndrome de Sjögren, vasculitis ANCA $(+)$ o infecciones por virus $\mathrm{B}$ y $\mathrm{C}$ de la hepatitis, citomegalovirus, parvovirus B19, HTLV I y Epstein Barr ${ }^{3,4,8}$. También se han visualizado en trasplantes renales, miositis por cuerpos de inclusión, histiocitosis, gliomas, linfomas y sarcomas ${ }^{4,9}$. En una revisión de 2.472 biopsias renales se encontraron ITR en células endoteliales glomerulares en 104 (4,2\%); de ellos, 81\% eran portadores de LES o una infección viral ${ }^{8}$.

En las biopsias de pacientes con infecciones por VIH se observan casi siempre ITR, incluso antes de que aparezcan manifestaciones clínicas o se haya hecho el diagnóstico de la infección ${ }^{2}$, como lo fue en este caso. En estos pacientes, además de observarse ITR en el endotelio glomerular, se las ha encontrado en el endotelio de los capilares peritubulares intersticiales, lo que parece ser característico de la infección por $\mathrm{VIH}^{10}$. Cuando se observan ITR en BpR que presentan una glomerulopatía colapsante, el diagnóstico de infección por VIH es prácticamente seguro ${ }^{10}$. A esta nefropatía se denomina "HIVAN" (HIV associated nephropathy). En ella, las ITR se observan en $80-90 \%$ de los casos ${ }^{9}$. También se han encontrado con escasa frecuencia ITR en BpR de pacientes con otras glomerulopatías, como enfermedad de Berger, enfermedad de cambios mínimos (ECM) y nefropatía membranosa. En las dos últimas, la presencia de ITR debe hacer sospechar una etiología secundaria ${ }^{8}$.

En este paciente, la existencia de hematuria microscópica persistente durante 14 años con ausencia de alteraciones en las imágenes renales, disfunción renal, hipertensión arterial y proteinuria, hizo plantear el diagnóstico clínico de EMBD. Apoyaban dicha sospecha la ausencia de antecedentes familiares de insuficiencia renal y la presencia de hematuria microscópica persistente en su madre. La EMBD es un trastorno frecuente en la población (5-9\%), también llamado "hematuria familiar benigna" y tiene un patrón genético de herencia autosómica dominante ${ }^{11}$. Se caracteriza por la presencia de hematuria microscópica aislada persistente o intermitente. Ocasionalmente pueden presentarse episodios de hematuria macroscópica. La aparición de proteinuria es infrecuente y rara vez se asocia a deterioro de la función renal ${ }^{12}$.

En este paciente, si bien la velocidad de filtración glomerular no se redujo, la aparición de proteinuria importante durante su evolución hizo plantear que tal vez no se tratara de una EMBD, sino de otra entidad. Por ello se realizó una BpR. Ella confirmó la sospecha clínica de EMBD (Figura 1) y mostró glomérulos hipertróficos compatibles con hiperfiltración glomerular, situación frecuente de observar en sujetos con resistencia a la insulina o gran masa corporal (desproporción entre masa corporal/masa renal) ${ }^{13,14}$. También, la ultraestructura mostró fusión pedicelar muy extensa, lo que explica la magnitud de la proteinuria. Esta alteración no es propia de la EMBD sino que se observa característicamente en formas primarias de enfermedades podocitarias ${ }^{15}$. En este paciente es planteable, dada su gran masa corporal, que esta alteración podocitaria sea una manifestación incipiente de una glomeruloesclerosis focal y segmentaria (GEFS) secundaria a su desproporción entre masa corporal/masa renal.

Otra explicación es que la lesión podocitaria responsable de la proteinuria sea secundaria a la infección por VIH. Característicamente, esta infección produce una glomerulopatía colapsante que origina proteinuria masiva y reducción de la velocidad de filtración glomerular, llevando a la falla renal en pocos meses ${ }^{16}$. Para el desarrollo de esta nefropatía se requiere, además de la infección 
de las células epiteliales por VIH, tener susceptibilidad genética y expresar algunos genes del virus en las células renales infectadas ${ }^{17}$. La HIVAN se observa casi exclusivamente en individuos de raza negra y en enfermedad por VIH avanzada. Por lo tanto, concluimos que ésta no fue la causa de proteinuria de nuestro caso, ya que el paciente carecía de todas estas condiciones mencionadas.

En las BpR de pacientes con sospecha de HIVAN, en $40 \%$ se han encontrado otros patrones histológicos como glomerulonefritis mesangiocapilar, glomerulonefritis por complejos inmunes, amiloidosis, nefropatía diabética, nefropatía membranosa, enfermedad de Berger y GEFS clásica. El rol del VIH en la patogénesis de estas glomerulopatías es desconocido y parece ser sólo coincidente ${ }^{18}$.

En este paciente, es planteable que las alteraciones pedicelares visualizadas en la $\mathrm{BpR}$ puedan ser secundarias a una forma menos definida de daño podocitario asociado a VIH en concurrencia con probables lesiones adaptativas por hipertrofia e hiperfiltración glomerular, donde no podemos descartar una forma incipiente o en remisión parcial intercurrente de una podocitopatía como GEFS-ECM.

El propósito de esta comunicación es advertir que frente al hallazgo incidental de ITR en el endotelio glomerular en $\mathrm{BpR}$, debe sospecharse la posibilidad de una infección por VIH y buscarse dirigidamente.

\section{Referencias}

1. Braun DL, Kouyos RD, Balmer B, Grube C, Weber R, Günthard HF. Frequency and spectrum of unexpected clinical manifestations of primary HIV-1 infection. Clin Infect Dis 2015; 61: 1013-21.

2. Kostianovsky M, Kang YH, Grimley PM. Disseminated tubule-reticular inclusions in acquired immunodeficiency syndrome (AIDS). Ultrastruct Pathol 1983; 4: 331-6.

3. Bromfield M, McQuillan R, John R, Avila-Casado C. The significance of tubuloreticular inclusions as a marker of systemic stimulation by interferons in a case of focal and segmental glomerulosclerosis associated with cytomegalovirus (CMV) infection. Clin Kidney J 2014; 7: 174-8.

4. Schaff Z, Heine U, Dalton AJ. Ultramorphological and ultracytochemical studies on tubuloreticular structures in lymphoid cells. Cancer Res 1972; 32: 2696-706.

5. Maturi RK, Font RL. Ultrastructural features and prevalence of tubuloreticular structures in the ocular vasculature of patients with AIDS: a study of 23 cases. Br J Opthalmol 1996; 80: 252-5.

6. Luu JY, Bockus D, Remington F, Bean MA, Hammar SP. Tubuloreticular structures and cylindrical confronting cisternae: a review. Hum Pathol 1989; 20: 617-27.

7. Grimley PM, Rutheford NM, Kang YH, Williams T, Woody JN, Silverman RH. Formation of tubuloreticular inclusions in human lymphoma cells compared to the induction of 2'-5'-oligoadenylate synthetase by leucocyte interferon in dose-effect and kinetic studies. Cancer Res 1984; 44: 3480-8.

8. Lee CJ, Suh KS, Kim KH, Chang YK, Na KR, Lee KW. The clinicopathologic significance of endothelial tubuloreticular inclusions in glomerular diseases. Ultrastructural Pathology 2013; 37: 386-94.

9. Marquart KH. Occurrence of tubuloreticular structures and intracisternal paracrystalline inclusions in endothelial cells of tissue fron different epidemiological types of Kaposi's sarcoma. Ultrastruct Pathol 2005; 29: 85-93.

10. Laurinavicius A, Rennke HG. Collapsing glomerulopathy: a new pattern of renal injury. Semin Diagn Pathol 2002; 19: 106-15.

11. Tryggvason K, Patrakka J. Thin basement membrane nephropathy. J Am Soc Nephrol 2006; 17: 813-22.

12. Savige J, Rana K, Tonna S, Buzza M, Dagher H, Wang YY. Thin basement membrane nephropathy. Kidney Int 2003; 64: 1169-78.

13. De Cosmo S, Menzaghi C, Prudente S, Trischitta V. Role of insulin resistance in kidney dysfunction: insights into the mechanism and epidemiological evidence. Nephrol Dial Transplant 2013; 28: 29-36.

14. Kwakernaak AJ, Toering TJ, Navis G. Body mass index and body fat distribution as a renal risk factors: a focus on the role of renal haemodynamics. Nephrol Dial Transplant 2013; 28 Supp 4: iv42-9.

15. Bagchi S, Agarwal S, Kalaivani N, Bhowmik D, Singh G, Mahajan S, et al. Primary FSGS in nephritic adults: clinical profile, response to immunosuppression and outcome. Nephron 2016; Jan 23.

16. Laurinavicius A, Hurwitz S, Rennke HG. Collapsing glomerulopathy in HIV and non-HIV patients: a clinicopathological and follow-up study. Kidney Int 1999; 56: 2203-13.

17. Wyatt CM, Klotman PE. HIV-1 and HIV-Associated nephropathy 25 years later. Clin J Am Soc Nephrol 2007; 2: S20-4.

18. Berliner AR, Fine DM, Lucas GM, Rahman MH, Racusen LC, Scheel PJ, et al. Observations on a cohort of HIV-infected patients undergoing native renal biopsy. Am J Nephrol 2008; 28: 478-86. 